Cahiers « Mondes anciens »

ANCIENS

Histoire et anthropologie des mondes anciens

$2 \mid 2011$

Journées doctorales ANHIMA 2008 et 2009

\title{
Espaces et identité : les colonies lusitaniennes du conventus emeritensis
}

Susana Marcos

\section{OpenEdition}

Journals

Édition électronique

URL : http://journals.openedition.org/mondesanciens/568

DOI : 10.4000/mondesanciens.568

ISSN : 2107-0199

Éditeur

UMR 8210 Anthropologie et Histoire des Mondes Antiques

Référence électronique

Susana Marcos, «Espaces et identité : les colonies lusitaniennes du conventus emeritensis », Cahiers "Mondes anciens » [En ligne], 2 | 2011, mis en ligne le 20 juillet 2011, consulté le 19 avril 2019. URL : http://journals.openedition.org/mondesanciens/568;DOI : 10.4000/mondesanciens.568

Ce document a été généré automatiquement le 19 avril 2019

\section{(c) (i) (9)}

Les Cahiers «Mondes Anciens » sont mis à disposition selon les termes de la licence Creative Commons Attribution - Pas d'Utilisation Commerciale - Pas de Modification 4.0 International. 


\title{
Espaces et identité : les colonies lusitaniennes du conventus emeritensis
}

\author{
Susana Marcos
}

1 Espace géographiquement périphérique de l'Occident romain, la province de Lusitanie, très vraisemblablement créée entre 16 et 13 av. J.-C., apparaît comme une référence dans les sources classiques en raison de la magnitudo agrorum de sa capitale, Augusta Emerita, l'actuelle Mérida' ${ }^{1}$. Néanmoins, loin d'être uniforme, la fondation de colonies est fonction de la volonté des autorités romaines : elle est le sceau de la réorganisation impériale, l'affirmation visuelle de l'occupation de l'espace. Néanmoins, si la colonisation est un phénomène ponctuel issu de la conquête, c'est surtout un processus important qui s'inscrit dans la durée et dont l'appropriation de l'espace marque le premier jalon. Dès lors, l'espace devient territoire. Cette notion sous-entend également que celui-ci doit être délimité et partagé, à la fois de façon géographique, à différentes échelles, mais aussi entre les différents occupants de cet espace. Toute colonisation implique en effet l'installation et la cohabitation, du moins théorique, des nouveaux arrivants avec les peuples précédemment installés en ces lieux dans un cadre lui aussi nouveau, symbole par excellence de la romanitas.

2 Ma thèse traitant des relations entre les cités de Lusitanie au Haut-Empire, j'ai choisi de me consacrer ici au territoire de la capitale, plus particulièrement la zone nord-est. Toutefois, si les sources sur cet espace sont importantes, il n'en est pas de même sur les liens entretenus avec ses voisines: les textes des auteurs gromatiques, l'épigraphie ainsi que parfois l'archéologie ne nous donnent la plupart du temps que des indices indirects. Or, la mise en place d'une véritable hiérarchie, à la fois sociale et spatiale, nous invite à réfléchir sur les notions de délimitations, de liens et de concurrence, et met en exergue l'aspect fondamental du concept de frontière coloniale, à la fois administrative, visuelle et volontairement marquée, mais aussi vécue. Ainsi, s'intéresser au processus de colonisation à travers l'œil du géographe, c'est tenter de comprendre la manière dont les 
sociétés qui vivent en ces lieux depuis plus ou moins longtemps façonnent l'espace selon leurs propres représentations.

3 C'est cette perception qu'ont les populations de leur espace, à la fois visible dans le paysage et sensible dans le vécu, qui est révélatrice de leur propre identité et nous donne des clés de lecture pour comprendre comment s'est construit l'espace colonial et percevoir s'il est le reflet d'une identité particulière.

4 Travailler sur le rapport qui existe entre l'espace et l'identité à l'échelle coloniale, c'est avant tout s'intéresser aux liens spatiaux, politiques, sociaux mais aussi culturels qui unissent la cité et sa pertica. Or, s'approprier un espace, en faire son territoire, c'est d'abord le conquérir, pour ensuite l'organiser, et par conséquent le délimiter. Par cette opération, un espace, dont la définition n'est jusque-là que géographique, est élevé au statut de territoire, c'est-à-dire un espace que l'on s'est approprié et où se reflète une identité particulière. Grâce aux auteurs gromatiques, le territoire éméritain ${ }^{2}$ est assez bien connu car il fait figure d'exemplum en raison de ses dimensions peu communes. Hygin le Gromatique (p. 136, 1-10) ${ }^{3}$ tout d'abord, nous apprend que les centuries sont de 400 iugera, avec un decumanus de 40 actus $^{4}$ et un kardo de 20. Par ailleurs, Frontin (repris par Agennius Urbicus, Th., p. 44, 5) témoigne pour sa part de trois points essentiels. La pertica, territoire initialement délimité et centurié, débordait de part et d'autre de l'Anas, actuel Guadiana $^{5}$. Ainsi le fleuve ne constitue pas une frontière, qui séparerait ici la Lusitanie de la Bétique voisine. De plus, malgré l'ampleur de l'ager centurié, les assignations se font à partir de la périphérie et non du centre de la civitas. Enfin, malgré une deuxième puis une troisième assignation, toutes les terres n'ont pas été distribuées : il y a donc des espaces « vides ", constitués de bois, de pâtures, etc... Par conséquent, cette construction spatiale nous permet de percevoir la conception romaine de l'espace, l'appropriation du territoire et son organisation, qui est pensée et réfléchie. En ce sens, l'attribution des terres par les extrémités du territoire n'est pas anodine, mais correspond bien à une volonté de jalonner l'espace, d'affirmer la propriété, d'imposer les nouvelles limites de la colonie à travers le paysage dans lequel évolue chaque habitant au quotidien.

Le contexte dans lequel a été créée la colonie et les motivations du choix de ce site vont également en ce sens. C'est en effet lors de la pacification après les combats contre les Astures et les Cantabres dans le Nord-Ouest hispanique que P. Carisius, légat d'Auguste propréteur, fonde la cité. L'empereur veut récompenser les vétérans de la $\mathrm{V}^{\mathrm{e}}$ légion Alaudae et de la $\mathrm{X}$ e Gemina. Toutefois, l'établissement d'une colonie se fait traditionnellement sur le territoire nouvellement conquis dans le but de bien marquer la victoire. Au contraire ici, Auguste rompt complètement avec ce mode de fonctionnement puisqu'il a cherché un endroit idéal pour fonder cette nouvelle cité. La topographie est avantageuse: le site est celui d'un large gué sur le Guadiana, à proximité de vastes étendues de terres propices à l'agriculture, et les dernières traces d'occupation humaine remontent au VIII ${ }^{\text {e }}$ s. av. J.- C. (MATEOS CRUZ 2001, p. 184-185). Qui plus est, c'est un espace jusqu'alors peu structuré, point qui a son importance pour la question de l'identité. Enfin, la nouvelle colonie présente l'avantage d'avoir une situation stratégique. Elle constitue un carrefour de voies de communications (Fig. 1), entre le fleuve d'une part et de l'autre l'important axe nord-sud qu'est la très ancienne Via de la Plata et qui met en relation l'Hispanie méridionale avec le Nord-Ouest. Si Augusta Emerita est conçue dès le départ comme un pôle d'attraction et d'influence, ces principaux axes sont autant de rayons qui vont permettre de développer de multiples réseaux avec les cités environnantes, à plus ou moins grande échelle, d'augmenter la présence romaine entre les cités de statuts 
privilégiés que sont Norba au nord, Metellinum à l'est et Pax Iulia à l'ouest, et de décupler d'autant la centralité de la capitale. Tous sont des marques de l'appropriation de l'espace et de sa structuration en fonction des besoins et des représentations des sociétés qui l'occupent.

Fig. 1 : Carte de localisation d'Augusta Emerita

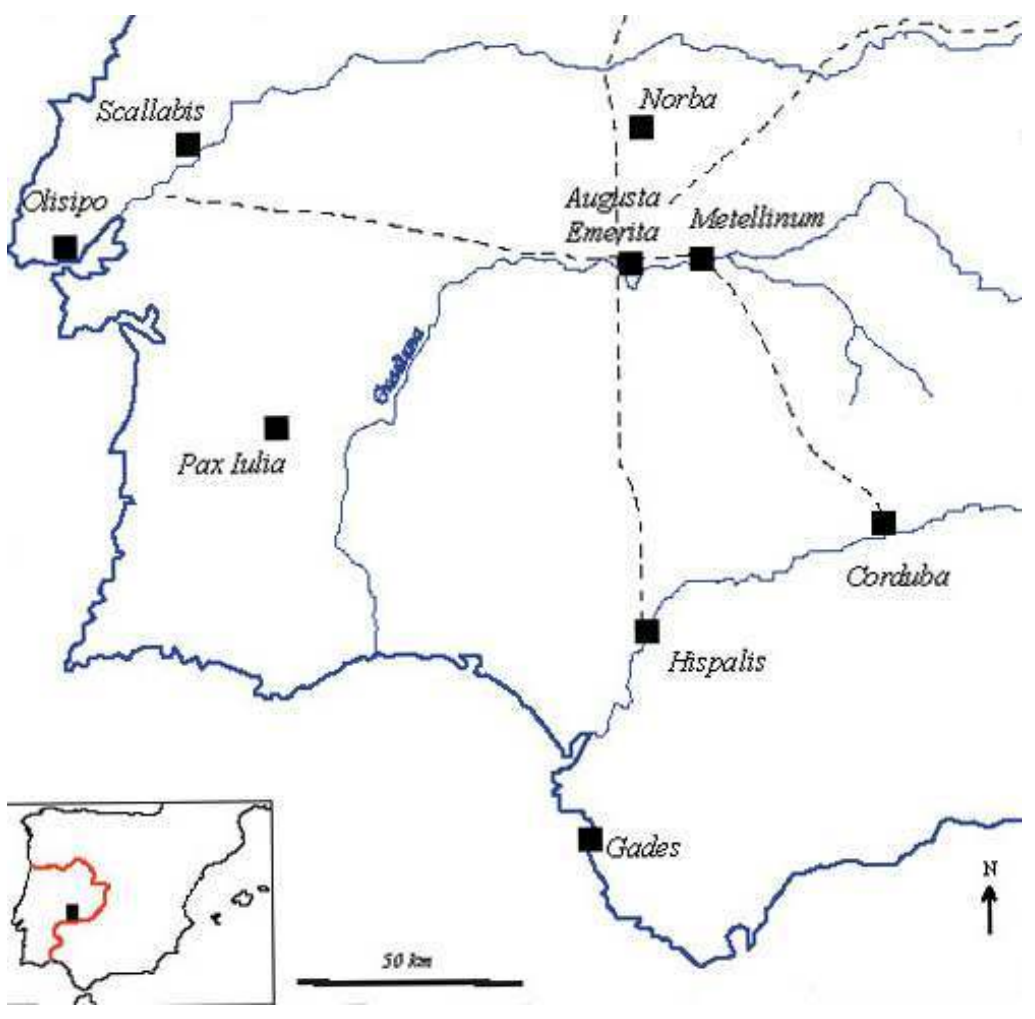

Carte Susana Marcos

6 Toutefois, la complexité du partage de l'espace ne saurait être comprise sans s'intéresser à la question fondamentale des confins du territoire colonial. Comme l'atteste une fois de plus Hygin (Th., p. 136, 1-10), il existe hors de la pertica d'Augusta Emerita proprement dite trois préfectures. Nous ignorons leur localisation, mais il est question, entre autres, d'une regio Turgaliensis qui devrait son nom à l'antique Turgalium (Trujillo, province de Cáceres). Pour P. LE ROUX (1999, p. 266 et 269), ces entités doivent être considérées soit comme un élargissement du territoire colonial, soit comme des ajouts de terres qui relevaient à l'origine d'une autre cité, elle indigène, qui s'est vue dessaisie d'une partie de son territoire au profit d'Augusta Emerita. Néanmoins, la cité en question garde son autonomie et le contrôle des terres qui ne lui ont pas été enlevées. Même s'il n'y a pas nécessairement continuité territoriale avec le sol colonial, la praefectura fait donc partie du territoire de la colonie : les terres assignées sont propriété des colons. La confiscation de terres est dans le cas d'Emerita d'autant plus surprenante que la pertica n'a pu être assignée dans sa totalité. Une question se pose alors: quelle est la nature des rapports entretenus entre la capitale romaine et les autres cités, notamment celles dont les terres sont prélevées? Au regard des sources dont nous disposons aujourd'hui, ce ne serait que spéculation de chercher à définir la localisation exacte de ces préfectures, et les causes de leur devenir. Toutefois, quelques pistes peuvent être abordées. 
7 D'après J.-C. SAQUETE CHAMIZO (2004, p. 385-386), la colonisation est trop souvent envisagée selon les schémas européens du XIX $\mathrm{x}$. qui font des colons les civilisateurs des peuples indigènes. Au contraire d'après lui, le phénomène de colonisation doit d'abord être conçu comme une punition. Certes, les soldats se voyaient attribuer un lot de terre dont ils jouissaient, mais est-il vraiment certain que ces hommes, qui ont combattu toute leur vie, étaient heureux de se convertir à une vie agricole du jour au lendemain? Vivaient-ils cette installation comme une réelle récompense pour leurs loyaux services? Qui plus est, ces attributions se font loin de leur terre natale, en un lieu qui leur est étranger. Si nous n'en avons pas la preuve pour Augusta Emerita, nous savons que certains colons revendaient leurs terres (SAQUETE CHAMIZO 2004, note 38). Enfin, ils ne bénéficient que de modestes parcelles : il y a peu de perspectives de richesses, et ils sont par ailleurs tenus de travailler un certain nombre de jours à la construction de la colonie. Bien évidemment, cette interprétation ne peut remettre en cause la volonté augustéenne de récompenser ses soldats au terme d'une carrière dédiée à l'Empire, d'autant que l'accès à la propriété est assurément pour certains de ces vétérans un bien inestimable. Cette politique impériale a le double avantage de conforter la présence romaine en ces terres nouvellement conquises tout en assurant ses fidèles soldats de la benevolentia de l'empereur à leur égard.

8 Sans vouloir remettre en cause les mérites de ces réflexions que nous partageons en partie, cette interprétation nous semble quelque peu extrême. Toutefois, il est clair que la vision idéalisée de la colonisation doit désormais être nuancée, en prenant davantage en compte le vécu de ces vétérans. Dans le cas des préfectures éméritaines, la mesure punitive, à l'encontre des indigènes semble assez claire, comme s'il y avait eu une volonté d'arracher des terres à l'organisation préexistante. Ici la colonisation est punition à l'égard des communautés qui vivaient à cet endroit, car la création coloniale modifie toutes les structures et les modes de vie qui avaient prévalu jusqu'à présent. En effet, ce n'est pas tant l'arrivée de populations nouvelles que la modification et l'abandon des formes de vie ancestrales au profit de schémas romains bien connus qui bouleversent les populations locales et leurs modes de vie. Mais quelles raisons pourraient justifier une telle attitude? Les sources sont insuffisantes et ce n'est là qu'une hypothèse, mais il est vraisemblable que ce soit avant tout une mesure politique prise à l'encontre de Turgalium, cité indigène ancienne sur laquelle la colonie aurait voulu prendre et marquer symboliquement son emprise.

9 Pour autant, cela signifie-t-il qu'à l'échelle des territoires qui dépendent de la colonie il existe une marque d'identité coloniale qui serait reconnue, voire affirmée? En réalité, l'étude de l'espace et celle des questions identitaires se mêlent et se nourrissent mutuellement pour aider à la compréhension de l'une et l'autre. En ce sens, le cas du territoire d'Augusta Emerita est assez significatif car il affiche une certaine unité. En effet comme le fait remarquer et l'analyse B. GOFFAUX (2006, p. 55-57), la cité se distingue par l'utilisation d'une datation anno coloniae, marqueur du rythme de vie propre à la colonie romaine, que l'on retrouve sur quatre inscriptions urbaines (ABASCAL PALAZÓN, 2002, p. 285-286). Or, cette spécificité a également été retrouvée sur une inscription découverte en remploi à Trujillo qui, malgré la difficulté du texte, indique sur le côté droit: Anno/c (oloniae) CCI/III ${ }^{6}$. Etant donné que l'on ne retrouve ce comput qu'à Emerita, il est tentant d'établir un lien particulier entre Turgalium et la capitale. L'ancienne cité indigène appartiendrait-elle à la regio Turgaliensis éméritaine ? Cette hypothèse permettrait alors de délimiter de façon plus précise l'espace de la praefectura. De fait, l'utilisation du 
calendrier colonial à la fois dans la cité capitale et sur un territoire qui lui est géographiquement extérieur marque bien sa dépendance administrative, mais aussi la reconnaissance, du moins officielle si elle n'est pas identitaire, du cadre colonial au sein duquel s'effectue la démarche ${ }^{7}$.

Certains aspects culturels, notamment religieux, viennent éclairer ces questions territoriales et identitaires. Comme en bien d'autres endroits, il existe autour de la capitale lusitanienne des divinités topiques (GOFFAUX 2006, p. 70), qui portent dans leur nom l'adjectif correspondant au lieu, ce qui est en soi un signe de territorialisation de la divinité. Mais c'est surtout le culte rendu à Lacipaea qui est particulièrement évocateur du lien étroit entre espace et identité. Nous connaissons une inscription ${ }^{8}$ à caractère votif, datée de 58-59, retrouvée dans la capitale sur une plaque de marbre blanc, de taille relativement imposante ${ }^{9}$. Peut-être cette divinité « indigène » a-t-elle bénéficié d'un culte public dans la cité ? Par ailleurs, il existe aussi une mansio nommée Lacipea ${ }^{10}$, située sur l'une des voies reliant la capitale à Caesaraugusta. Or, c'est dans cette zone qu'ont été retrouvés trois autels de granit dédiés à cette déesse ${ }^{11}$, seules autres attestations que nous en ayons. Par conséquent, Lacipaea est bien une divinité régionale ancestrale, adoptée par les Romains dès l'époque julio-claudienne, honorée à la fois dans le chef-lieu de la colonie, mais aussi sur son territoire, géographiquement plus éloigné. Ici, la relation entre la colonie et la praefectura qui en dépendait est claire et explicite. Même si la regio Turgaliensis garde ses caractéristiques propres, elle est nettement soumise à l'influence d' Augusta Emerita. En ce sens, l'exemple de ce culte est particulièrement illustratif du lien qui peut unir espace et identité, cité et territoire.

11 Le processus de colonisation, et par conséquent les partages spatiaux qui en découlent, pose tout naturellement la question des rapports entretenus avec les cités voisines. Or, la répartition des colonies en Lusitanie est particulièrement inégale. Il n'en existe que cinq dont Scallabis au nord et Pax Iulia à l'ouest, toutes deux capitales de conventus, puis Augusta Emerita, capitale de province, dont le territoire jouxte vraisemblablement celui de deux colonies plus anciennes : Norba et Metellinum. Dès lors, cette concentration particulière justifie que l'on s'interroge sur plusieurs aspects, au premier rang desquels les limites territoriales et la question des frontières. En effet, comment s'est construit le territoire voisin face à la création de la capitale ? A-t-il été repoussé par les limites de la colonie éméritaine ou y a-t-il eu au contraire prise en compte des populations locales et de l'organisation préexistante? En raison du manque de sources, c'est une question à laquelle il est très délicat de répondre. Tout d'abord, il n'y a pas de document faisant référence aux termini posant les limites entre les trois colonies. De même, aucune source littéraire ne mentionne par exemple un ager ou des communautés rurales qui auraient pu vivre sur ces terres. Par ailleurs, nous ne disposons que de peu de matériel numismatique: des trois cités, seule Augusta Emerita a eu le droit de battre monnaie. Pourtant, parmi ces frappes, aucune référence n'est faite à cette question. Enfin, l'étude de la distribution de la tribu Sergia, qui est à la fois celle de Metellinum et de Norba, alors que les citoyens de la capitale font partie de la Papiria, ne peut être fructueuse : une seule attestation est connue pour la première (HABA QUIRÓs, 1998, p. 281).

Toutefois, l'étude de l'organisation de l'espace, notamment grâce aux traces de cadastration, a pu démontrer les caractéristiques propres de chacun de ces territoires: l'analyse de l'occupation rurale est en soi très instructive. Sur le territoire étudié au sud d'Augusta Emerita, il existe une nette homogénéité du peuplement lié aux facteurs géologiques et topographiques, suivant l'orientation de la centuriation, elle-même de 
distribution très régulière ${ }^{12}$. À Norba, même si les traces sont plus rares, le peuplement rural est important et uniforme, mais en des zones bien délimitées (ALONSO SÁNCHEZ, CERILLO M. DE CÁCERES, FERNÁNDEZ CORRALES 1994, p. 81), c'est-à-dire là où les terres sont les meilleures, au sud de la cité. D'après l'archéologie, les établissements ruraux sont ici à une distance moyenne de $3 \mathrm{~km}$ les uns des autres: on estime alors la superficie des propriétés à environ 800 ha. Hors de cette zone restreinte, la distance entre ces installations est plus grande, environ $5 \mathrm{~km}$, car les sols sont plus pauvres. Le modèle théorique des exploitations serait donc de 1600 ha en moyenne sur le territoire de Norba, tout comme celui de Turgalium (CERILLO MARTíN DE CÁCERES et FERNÁNDEZ CORRALES 1980, p. 157-175), ce qui diffère de la grande zone agricole éméritaine des Tierras de Barros ${ }^{13}$. Même s'il y a une influence de l'un sur l'autre, les modèles économiques des colonies respectives sont donc bien distincts. La même remarque est valable pour Metellinum (HABA QUIRós 1998, p. 283-285) où l'on peut observer grâce à la photographie aérienne militaire de 1956, à l'est des parcelles plus vastes en raison du relief montagneux, tandis qu'au sudest, en l'absence d'obstacles naturels, les dimensions sont plus modestes, suivant la voie Emerita/Corduba. De même pour Norba, la limite avec la capitale est la barrière nette que constitue la Sierra ${ }^{14}$.

13 Ainsi, l'influence de la cité serait également fonction de la plus ou moins grande proximité d'éléments de relief. C'est cet autre élément qui apparaît comme tout à fait primordial dans la délimitation des territoires respectifs car ce sont des signes remarquables, visibles dans le paysage, marqueurs du territoire et de l'identité. Finalement, malgré le manque de sources directes, des limites relativement viables semblent apparaitre, même s'il est tout à fait possible qu'il y ait eu des réajustements locaux qu'il nous est impossible de vérifier à l'heure actuelle. N'oublions pas que lors de la déduction de la colonie d'Augusta Emerita en 25 av. J.-C., l'Hispanie est constituée de deux provinces : la Citérieure et l'Ultérieure dont la limite passe à l'est de Metellinum (LE ROUX 1999, p. 272). Le passage de deux à trois provinces, avec la création de la Lusitanie, a donc pu entraîner une réorganisation locale à partir de critères qui nous échappent encore pour le moment.

14 L'étude géographique et cartographique permet de mieux apprécier quel fut le poids de chacune des cités, Augusta Emerita, Norba et Metellinum, et quel type de rapports les liaient les unes aux autres. Dans son étude sur les tribus romaines, G. FORNI (2006, p. 169-184) tentait de délimiter le territoire d'Augusta Emerita à partir de la diffusion de la Papiria qui est celle des citoyens de la capitale lusitanienne ${ }^{15}$. En Hispanie, seules deux colonies, Emerita et Astigi en Bétique, sont inscrites dans cette tribu. Toutefois, comme le fait remarquer P. LE ROUX (1999, p. 271), cela ne peut correspondre aux limites territoriales exactes : cette répartition dessine davantage une aire d'influence de la capitale à travers les déplacements de ses citoyens, témoin des liens territoriaux de la colonie qui nous intéressent. Comme nous pouvons le constater sur la carte élaborée par G. FORNI (Fig. 2), la diffusion de la tribu Papiria hors du territoire éméritain est assez vaste : elle déborde à la fois à l'ouest, sur les territoires des lointaines cités d'Ammaia et Ebora (la région est d'ailleurs considérée comme une possible zone de préfecture), au sud en territoire bétique, et au nord et nord-est de Mérida, visiblement sous l'influence de Norba et Turgalium. 
Fig. 2 : Répartition des attestations de la tribu Papiria à proximité du territoire d'Augusta Emerita (d'après G. Forni)

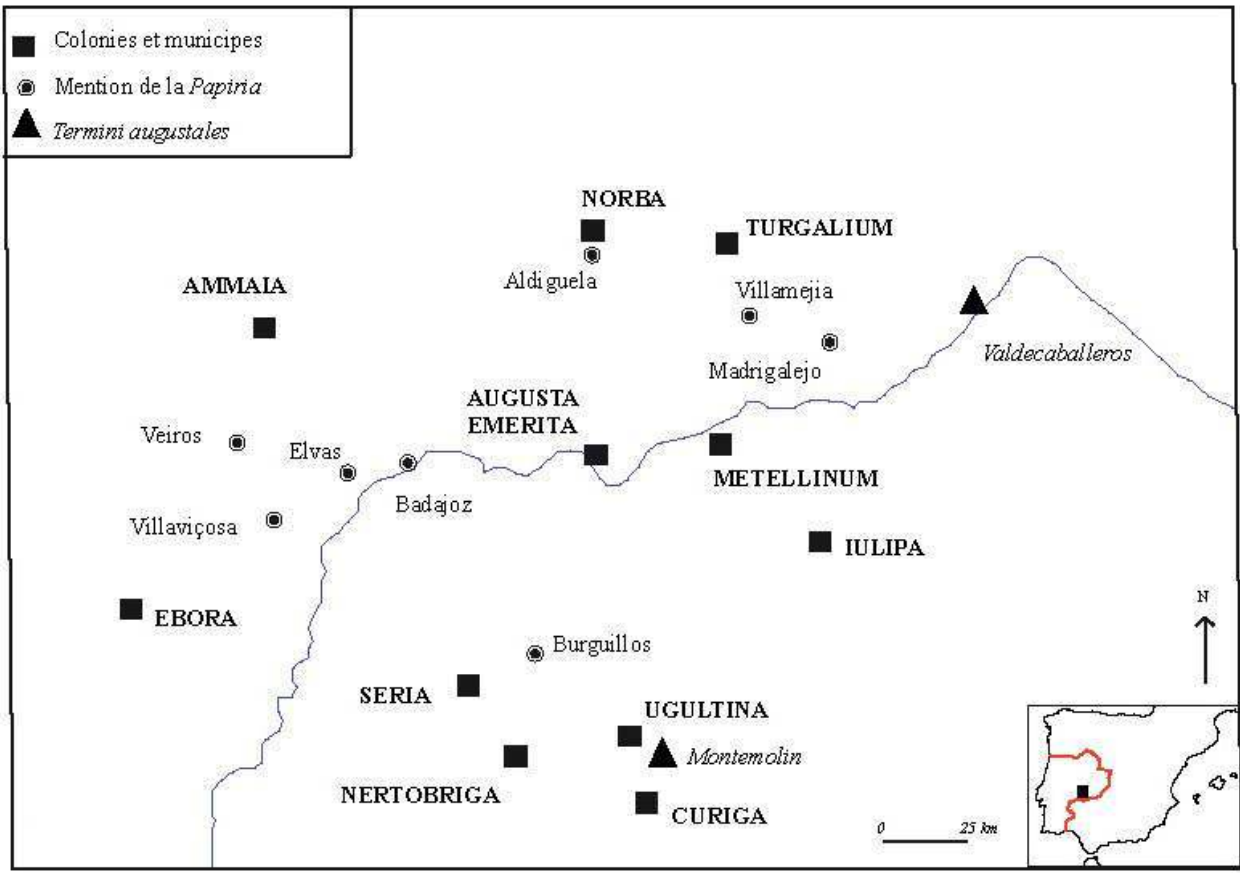

Carte Susana Marcos

Si au contraire nous essayons de considérer l'attraction que la capitale peut exercer sur les cités voisines, il est évident qu'elle apparaît comme un véritable pôle attractif. L'exemple de la gens Norbana, issue du nom du fondateur éponyme de la cité, C. Norbanus Flaccus, et parmi laquelle comptent quelques membres de l'élite locale ${ }^{16}$, est particulièrement révélateur quoique hypothétique ${ }^{17}$. Bien que présents ailleurs, les porteurs de ce nomen se concentrent comme nous pourrions nous y attendre dans un noyau au sud-est de la Lusitanie, autour de Norba ${ }^{18}$. Néanmoins à l'échelle locale, l'analyse peut être affinée. Sur la totalité des inscriptions, seulement cinq proviennent de la civitas même : les autres ont été retrouvées sur son territoire ${ }^{19}$. En parallèle, c'est du sud de la région que provient la grande majorité des documents. Treize inscriptions ont été trouvées dans la localité moderne d'Ibahernando, où se situerait la regio Turgaliensis; quatre autres appartiennent à l'ancienne Turgalium et à ses environs, espace qui, comme nous l'avons vu, dépendait probablement de la capitale; et enfin, sept inscriptions ont été retrouvées dans la cité-capitale ${ }^{20}$. À la lumière de ces indices, l'influence d'Augusta Emerita sur toute la région ne peut faire de doute, et ce y compris à partir de ses terres préfectorales, qui pourtant sont plus proches géographiquement de la colonie de Norba.

L'étude des magistratures de ces colonies (Fig. 3 ; cURCHIN 1990) conforte cette hypothèse. Au-delà des aléas des découvertes des inscriptions et de l'usage épigraphique, reflet des pratiques locales, la différence de statut administratif pourrait en partie expliquer que le nombre de magistrats attestés à Metellinum et Norba soit inférieur à celui de la capitale, mais bien plus encore que ces personnages soient de moindre envergure. S'il est évident que des magistrats sont élus tous les ans dans ces cités, cela laisse toutefois l'impression d'une sorte d'effacement des deux colonies préexistantes, impression accentuée par le 
manque d'investissement des deux cités dans le culte impérial. De fait, à ce jour, nous ne connaissons qu'un seul augustal originaire de Norba (SAQUETE CHAMIZO, 1997, p. 146-147), fonction le plus souvent desservie par des affranchis. Cela est d'autant plus surprenant que l'exercice de ce culte est prestigieux et particulièrement recherché par l'élite lusitanienne, comme en témoigne l'activité de cités comme Ammaia, Ebora ou Olisipo, qui plus est de statut juridique inférieur par rapport à Norba ou Metellinum. De la même manière, le nombre d'évergésies attestées dans ces deux dernières cités est bien moindre par rapport à la capitale. Dans une même zone géographique, nous pourrions penser qu'il existe un même epigraphic habit. Pourtant, sur la base des travaux de J. ANDREU PINTADO (2004, p. 207-220), on dénombre seulement deux inscriptions provenant de Metellinum et deux autres de Norba ${ }^{21}$, alors que leur nombre est décuplé pour Augusta Emerita. Une fois de plus, cela donne l'image d'un certain retrait politique qui reflète sans doute un dynamisme urbain moindre.

Fig. 3 : Nombre d'attestations de magistrats par cité

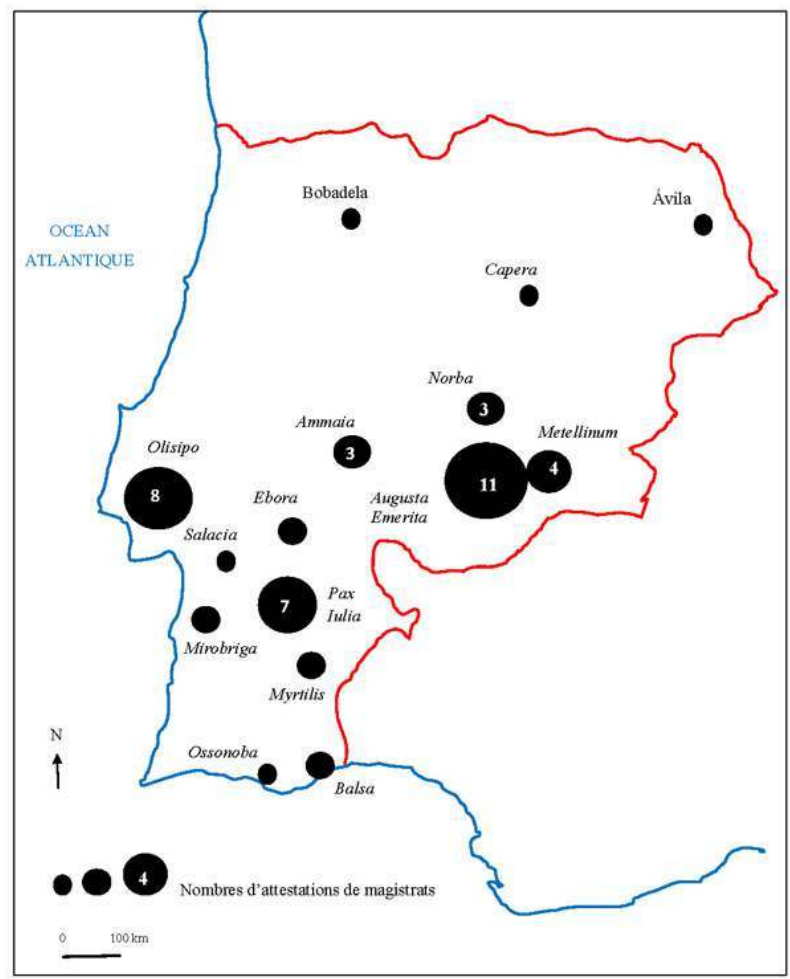

Carte Susana Marcos

17 Les deux colonies seraient-elles lésées par la trop grande proximité géographique de la capitale? Se pose alors la question de la «macrocéphalie » éméritaine : n'y aurait-il pas une emprise démesurée de la capitale sur le reste du réseau urbain, notamment sur Norba et Metellinum qui ont a priori le même statut colonial?

«Foyer politique, économique et culturel, la ville agit avec force sur la structuration du territoire qui l'environne, poussant d'autant plus loin son influence que le poids des fonctions qu'elle exerce est grand». Voilà comment J.-G. GORGES (1990, p. 92) décrit la situation d'Augusta Emerita. Néanmoins, la supériorité hiérarchique, politique et administrative de la capitale aurait-elle été un facteur de déclin pour les deux autres colonies à proximité ? Pour Norba, cela semble vérifié : la cité est un « centre secondaire » 
(GORGES 1990, p. 99), peuplé de riches propriétaires terriens, à l'ombre de la capitale. Dans le cas de Metellinum, cela est davantage débattu (HABA QUIRós 1998, p. 295 et note 64, s'oppose à GORGES). Il est évident que les deux colonies ont joué un rôle moindre (cf. Fig. 2 et 3), ne serait-ce qu'en raison du poids administratif que représentent les statuts de capitale de province d'une part et de conventus par ailleurs, et des multiples fonctions (administratives, religieuses, judiciaires,...) qui y sont liées. Par conséquent, comme nous venons de le constater, la concentration de magistrats y est forte alors que relativement peu sont connus ailleurs. Tout cela conforte bien évidemment le rôle central de la cité dans le culte impérial, auquel les colonies de Metellinum et Norba ne semblent que très peu associées. Qui plus est, la capitale est un lieu de représentation du pouvoir impérial et de Rome. En cela, la monumentalité de la cité, les programmes iconographiques que l'on y trouve sont particulièrement importants. Au contraire, le décor architectural retrouvé à Norba et Metellinum, d'époque républicaine, n'a aucune mesure avec ce qui semble apparaître à Augusta Emerita, dont le simple nom affirme bien le rôle de l'empereur.

Enfin, la richesse agricole de la capitale a pesé d'un poids considérable, puisque la centuriation coloniale est en grande majorité située sur des terres très fertiles où se concentrent de nombreuses exploitations. J.-G. GORGES (1990, p. 91-113) ${ }^{22}$ démontre qu'un petit nombre de villae est le signe d'une moindre influence de la colonie. Or, en Lusitanie, il existe un véritable pôle Emerita/Norba/Turgalium avec deux indéniables concentrations autour d'Emerita/Metellinum mais aussi dans le sud-est de Norba/sud-ouest de Turgalium ${ }^{23}$. La première regroupe à elle seule environ un tiers des sites connus, datés du $\mathrm{I}^{\mathrm{er}}$ au $\mathrm{IV}^{\mathrm{e}} \mathrm{s}$. et qui mêlent caractère luxueux et agricole; la seconde se consacre essentiellement à la céréaliculture et à l'élevage. Certes, ces exploitations sont destinées à ravitailler les deux centres urbains les plus proches, mais elles appartiennent sans doute aussi à la sphère économique éméritaine. La représentation cartographique des villae sur le territoire civique traduit donc à la fois le poids respectif de chacune des cités, mais aussi leur emprise sur le territoire. Reste à savoir si ces territoires de Turgalium dépendaient effectivement de la capitale en tant que praefectura, ce qui accroitrait d'autant l'influence, voire la dépendance des villae de Norba par rapport au centre de gravité que représente la capitale.

Si les cités possèdent leur territoire propre, les multiples fonctions de la capitale entraînent sans doute le déclin des autres colonies qui l'avaient chronologiquement précédée, ou du moins les font entrer dans sa "sphère d'influence ", dessinant ainsi un vaste réseau urbain dont les pôles plus ou moins influents sont reliés par les rayons que représentent les voies de communications.

Le lien qui unit les notions d'espace et d'identité permet de comparer les différents modèles de colonisation dans cet espace lusitanien fort stratégique. Or, nous pouvons facilement constater que chacune des colonies correspond à une étape chronologique différente, adaptée aux besoins du moment et fonction des tactiques républicaines ou impériales. Comme l'a décrit la thèse de S. HABA QUIRós (1998, p. 248-250), il existe une continuité entre le site indigène antérieur et l'établissement romain de Metellinum. En effet, à l'époque préromaine l'habitat est situé sur un promontoire à plus de 300 mètres d'altitude qui domine visuellement un vaste territoire et l'un des gués les plus importants du fleuve Guadiana: la population se concentre sur la butte, entourée d'une enceinte fortifiée. C'est un noyau que l'on peut qualifier d'urbain, organisé d'un point de vue socioéconomique. On accepte généralement l'installation d'un camp romain à l'occasion de la guerre contre Sertorius ${ }^{24}$. Le site se développe et devient selon cette hypothèse une 
garnison permanente et un noyau de peuplement romain : la colonie romaine déborde audelà des murs. Ce serait sur le site indigène qu'est installé le praesidium, tandis que les monuments caractéristiques de la romanitas sont construits sur le reste de l'espace. Même si cette thèse est fortement remise en question faute de sources fiables pour l'époque républicaine (CADIOU 2008, p. 352-353), ce qui importe essentiellement est la continuité du site et la compréhension des critères de l'occupation de l'espace. Par conséquent, et contrairement à d'autres fondations, Metellinum serait d'abord un centre à vocation militaire, en raison de sa facile défense. La cité domine aisément le riche territoire agricole, le gué sur le fleuve et les voies de communication qui sont à proximité. C'est donc un point de contrôle idéal.

C'est en 35-34 av. J.-C. que C. Norbanus Flaccus, gouverneur d'Hispanie Citérieure, aurait fondé la Colonia Norba Caesarina. Le nom est à la fois à la mémoire de sa famille, originaire d'Italie, et une marque de témoignage à l'égard du triumvir Octavien César. Nous ne savons que peu de choses sur Norba ${ }^{25}$, mais selon toute vraisemblance, la cité s'est élevée sur les restes d'un castrum indigène situé à proximité d'une rivière et dominant la route qui reliait la Bétique avec le nord et le nord-ouest de la péninsule. À proximité existaient deux camps, Castra Servilia et Castra Caecilia ${ }^{26}$, dont la présence même témoigne de la volonté de contrôle et de mainmise sur ce territoire entre Tage et Guadiana au moment de la lutte contre les peuples de la région, Lusitaniens et Vettons. D'ailleurs, Castra Caecilia doit son nom à son fondateur, Q. Caecilius Metellus Pius, celui même qui a fondé à peu près à la même époque la colonie de Metellinum. Ce qui est particulièrement intéressant dans le cas de Norba du fait de la grande mobilité de l'armée républicaine, c'est qu'une fois la région conquise et dominée, les deux camps perdent leur fonction militaire initiale. L'existence historique antérieure de ces deux ensembles à la deductio de la colonie de Norba a sans doute justifié le processus de contributio (Pline, HN, IV, 117). Ces deux noyaux ${ }^{27}$, assimilables à des faubourgs de la cité, deviennent deux cités contributae dépendantes de la Colonia Norba Caesarina d'un point de vue juridique et administratif, peuplées à la fois de population locale, vraisemblablement d'origine vettone, et d'anciens vétérans de l'armée romaine, bénéficiant majoritairement de la citoyenneté romaine. De cette manière, la constitution d'un nouvel établissement de catégorie administrative supérieure qu'est Norba affecte sans doute la condition juridique des habitants des cités contribuées préexistantes : tous ceux qui y avaient la citoyenneté romaine sont assimilés en droit aux citoyens vivant dans la colonia, ce qui apparaît comme un bienfait de l'État romain. À cela il faut ajouter une attestation ${ }^{28}$ qui nous est parvenue et qui rappelle l'acte d'évergétisme d'un citoyen romain, sans doute notable local, en faveur d'un vicus au nom indigène. Cela prouve à la fois la prise en compte du substrat local, des réalités préexistantes aux Romains, et reconnaît également les identités locales et leurs particularités qui s'intègrent à leur tour aux schémas romains.

La création d'Augusta Emerita, postérieure à celle de Metellinum et Norba, est dès le début conçue comme le symbole et la représentante, du gouvernement impérial et de la romanité. Lors de la déduction en 25 av. J.-C., il n'existe aucune contrainte administrative stricte : la région semble peu peuplée et la réserve de terres importante ${ }^{29}$. De ce fait, la création ex novo de la capitale est traditionnellement acceptée ${ }^{30}$ et conforte l'organisation du territoire précédemment présentée, avec une cadastration très particulière. Même si le faible peuplement régional a été l'un des critères de sélection de cette région pour installer une nouvelle colonie, alors que deux autres y existaient déjà, nous ne pouvons vraisemblablement pas croire que la zone était totalement vide d'hommes. Cela pose alors 
la question de l'intégration des populations locales sur le territoire colonial. D'ailleurs, Strabon (Géographie, III, 2, 15) évoque la fondation de villes à population mixte en Hispanie, au nombre desquelles il compte Augusta Emerita, chez le peuple turdule ${ }^{31}$. Or, si les sources archéologiques sont inexistantes, l'épigraphie peut apporter une interprétation nouvelle. Dans la nécropole de "El rincón de Gila ", à une vingtaine de kilomètres de Badajoz, a été retrouvée une stèle de granit ${ }^{32}$, en assez mauvais état de conservation mais de mesures assez importantes, mentionnant un $C$ (aius) Allius/Tangini/f (ilius) Pap (iria)/[...]. Autrement dit, d'après l'onomastique, l'inscription fait référence à un personnage d'origine locale, né d'un père pérégrin ${ }^{33}$, et qui a reçu par la suite la citoyenneté romaine, comme l'atteste son inscription dans la tribu Papiria. Il y a donc bien eu intégration probable d'indigènes parmi les colons. À cela vient s'ajouter le fait que l'étendue du territoire d'Augusta Emerita implique nécessairement la présence d'éléments locaux. Comme y fait d'ailleurs référence la loi d'Urso (CIII), cette population vient s'ajouter aux vétérans d'origine italique de la colonie romaine et nourrit ainsi l'identité coloniale.

La question de l'espace est indissociable de la notion d'identité. À l'origine même du concept "d'espaces partagés», toutes deux s'éclairent mutuellement d'un regard nouveau. Les aspects géographiques de l'organisation de l'espace permettent de mieux comprendre quel visage les Romains ont voulu donner à ces terres conquises, comment ils se les représentaient, et comment ils se les sont appropriés, face à une identité locale que l'on tend trop souvent à considérer comme secondaire parce que peu présente dans les sources.

Dans cette étude, j'ai volontairement travaillé à de multiples échelles afin de tenter de comprendre de quel poids a joué la colonie sur son voisinage et dans des contextes divers. Il est indéniable qu'Augusta Emerita fait figure de pôle attractif au sein de cet espace. De toute évidence, il semble aujourd'hui prouvé que les populations locales ont été intégrées dans le processus de colonisation, même si ce fut parfois par la force. L'évolution chronologique semble même indiquer que la colonie a pu être dans certains cas un référent culturel, mais qui varie en fonction de l'échelle prise en considération. Quoi qu'il en soit, si les populations locales ont perdu leurs anciens repères, elles en ont sans doute trouvé d'autres ou fait évoluer les précédents vers des formes plus proches des nouveaux modèles apportés par les Romains. D'un point de vue administratif, la frontière coloniale fait, elle, vraiment sens. Elle est connue et reconnue. Elle est véritablement vécue et appropriée. Mais est-elle une référence identitaire adoptée par les populations? C'est pour le moment plus difficile à déterminer. Ce sont autant d'enjeux qui lient l'espace et l'identité dans le cadre de la colonisation de la Lusitanie et qui permettront sans doute à l'avenir d'ouvrir autant d'axes de recherche.

BIBLIOGRAPHIE 


\section{Abréviations}

AE = L'Année Épigraphique, Paris, 1888-.

CIL, II = E. HUBNER (éd.), Corpus InscriptionumLatinarum, vol. II, Berlin, 1869 ; Supplementum, 1892.

$E R A E=$ L. GARCÍA IGLESIAS, Epigrafía romana de Augusta Emerita, Madrid, 1973, thèse

dactylographiée.

EE, VIII = Ephemeris Epigrafica, 1872-1913.

HEp = Hispania Epigraphica, Universidad Complutense, Madrid, 1989- .

\section{Bibliographie générale}

(1976), Augusta Emerita. Actas del Simposio internacional conmemorativo del bimilenario de Mérida, Mérida.

ABASCAL PALAZÓN J. M. (2002), « Fasti consulares, fasti locales y horologia en la epigrafía de Hispania ", AEA 75, p. 269-286.

AGUILAR SÁENZ A., GUICHARD P., LEFEBVRE S. (1992-1993) « La ciudad antigua de Lacimurga y su entorno rural », Stud. hist. $H^{a}$ antig. 10-11, p. 109-130.

ALONSO SÁNCHEZ A., CERILLO M. DE CÁCERES E., FERNÁNDEZ CORRALES J. M. (1994), « Tres ejemplos de poblamiento rural romano en torno a ciudades de la Via de la Plata : Augusta Emerita, Norba y Capara ", dans Gorges J.-G. et Salinas de Frías M. éd., Les Campagnes de Lusitanie romaine, MadridSalamanque, p. 67-87.

ANDREU PINTADO J. (2004), Munificiencia pública en la provincia Lusitania (siglos I- IV d.C.), Zaragoza. ARIÑO E. et GURT J. M. (1994), « Catastros romanos en el entorno de Augusta Emerita. Fuentes literarias y documentación arqueológica », dans Gorges J.-G. et Salinas de Frías M. éd., Les Campagnes de Lusitanie romaine, Madrid-Salamanque, p. 45-66.

ARIÑO E. et GURT J. M. (1998), « Les centuriations d'Augusta Emerita », dans Clavel-Lévêque M. et Vignot A. éd., Atlas historique des cadastres d'Europe, vol. I, Dossier II, Luxembourg.

CADIOU FR. (2008), Hibera in terra miles. Les armées romaines et la conquête de l'Hispanie sous la République (218- 45 av.J.-C.), Madrid.

CALLejo SERRANo C. (1968), « La arqueologia de Norba Cesarina », AEA 41, n¹17 et 118, p. 121-149.

CANTO A. (1987), « Colonia Iulia Augusta Emerita : Consideraciones en torno a su fundación y territorio », Gerión 7, p. 149-205.

CERILLO MARTÍN DE CÁCERES E. et FERNÁNDEZ CORRALES J. M. (1980), « Contribución al estudio del asentamiento romano en Extremadura : análisis espacial aplicado al S. de Trujillo », Norba, 1, p. 157-175.

CURCHIN L. A. (1985), « Vicus et pagi in Roman Spain », REA LXXXVII, 3-4, p. 327-343.

CURCHIN L. A. (1990), The Local magistrates of Roman Spain, Toronto, 1990.

CURCHIN L. A. (1990b), «Élite urbaine, élite rurale en Lusitanie », dans Les Villes de Lusitanie romaine. Hiérarchies et territoires, Paris, p. 265-276.

ETIENNE R. (1995), « À propos du territoire d'Emerita Augusta (Mérida) », dans Clavel-Lévêque M. et Plana-Mallart R. éd., Cité et Territoire, Paris, p. 27-32. 
FORNI G. (2006), Le Tribù romane, IV : Scripta minora, Rome.

GOFFAUX B. (2006), «Formes d'organisation des cultes dans la Colonia Augusta Emerita (Lusitanie) », dans Dondin-Payre M. et Raepsaet-Charlier M.-Th. éd., Sanctuaires, pratiques cultuelles et territoires civiques dans l'Occident romain, Bruxelles, p. 51- 97.

GORGES J.-G. (1990), « Villes et villas de Lusitanie (Interactions-échanges-autonomies) », dans Les Villes de Lusitanie romaine. Hiérarchies et territoires, Paris, p. 91-113.

GORGES J.-G. (1993), « Nouvelle lecture du fragment de forma d'un territoire voisin de Lacimurga », MCV 29 (1), p. 7-23.

GORGES J.-G. et RODRÍGUEZ MARTÍN F. G. (2004), « Los territorios antiguos de Mérida. Un estudio del territorium emeritense y de sus áreas de influencia », dans Nogales Basarrate T. éd., Augusta Emerita. Territorios, Espacios, Imágines y Gentes en Lusitania Romana, p. 93-128.

GUILLAUMin J.-Y. (2005), Les arpenteurs romains. T I : Hygin le Gromatique, Frontin, Paris.

HABA QUIRós s. (1998), Medellín romano. La Colonia Medellinensis y su Territorio, Badajoz.

LE ROUX P. (1994), «Vicus et castellum en Lusitanie sous l'Empire », dans Gorges J.-G. et Salinas de Frías M. éd., Les Campagnes de Lusitanie romaine, Madrid-Salamanque, p. 151-160.

LE ROUX P. (1999), « Le territoire de la colonie auguste de Mérida. Réflexions pour un bilan », dans Gorges J.-G. et Rodríguez Martín F. G. éd., Économie et territoire en Lusitanie romaine, Madrid, p. 263-276.

MATEOS CRUZ P. (2001), « Augusta Emerita. La investigación arqueológica en una ciudad de época romana », AEA 74, p. 183-208.

NAVARRO CABALLERo M. et RAMÍrez SÁDABA J. L. éd. (2003), Atlas antroponímico de la Lusitania romana, Mérida-Bordeaux.

SÁEZ FERNÁNDEZ P. (1990), « Estudio sobre una inscripción catastral colindante con Lacimurga », Habis 21, p. 205-227.

SÁEZ FERNÁNDEZ P. (1992-1993), « Nuevas perspectivas en relación a la ordenación territorial del sur de la Lusitania romana ", Stud. hist. $H^{a}$ antig. 10-11, p. 99-108.

SALAS MARTíN J. (1996), « Fuentes para el estudio de la Colonia Norba Caesarina y sus contributa Castra Servili y Castra Caecilia », Anas 9, p. 59-78.

SÁNCHEZ BARRERO P. D. (2004), « La estructuración del territorio emeritense : la organización espacial del paisaje en las proximidades de la colonia », dans Nogales Basarrate T. éd., Augusta Emerita. Territorios, Espacios, Imágines y Gentes en Lusitania Romana, Mérida, p. 67-91.

SAQUETE CHAMIZO J.-C. (1997), Las Elites sociales de Augusta Emerita, Mérida.

SAQUETE CHAMIZO J.-C. (2004), « Territorios y gentes en el contexto histórico de la fundación de la colonia Augusta Emerita », dans Nogales Basarrate T. éd., Augusta Emerita. Territorios, Espacios, Imágines y Gentes en Lusitania Romana, Mérida, p. 373- 397.

SILLIÈRES P. (1990), « Voies romaines et limites de provinces et de cités de Lusitanie », dans Les Villes de Lusitanie romaine. Hiérarchies et territoires, Paris, p. 73-88.

WIEGELS R. (1976), « Zum Territtorium der augusteischen Kolonie Emerita », MM 17, p. 258-284. 


\section{NOTES}

1. Pour une vision globale des problématiques, voir Augusta Emerita (1976).

2. Nous n'entrerons pas ici dans les larges débats dont ce territoire a fait l'objet afin d'en déterminer les limites exactes. Pour un état de la question, cf. GORGES et RODRÍGUEZ MARTíN (2004), p. 95-98 et LE ROUX (1999), p. 263-264, et plus particulièrement la note 2.

3. Voir également l'édition commentée en français GUILLAUMIN (2005).

4. La jugère équivaut à un rectangle de 28800 pieds carrés, c'est-à-dire environ 25 ares. Un actus est une surface de 4 pieds de large sur 120 de long.

5. Sur ce point largement débattu, voir notamment SILLIÈRES (1990), p. 73-88; SÁEZ FERNÁNDEZ (1992-1993), p. 100-103 et Id. (1990), p. 206, 218-220; AGUILAR SÁENZ, GUICHARD, LEFEBVRE (1992-1993), p. 110-111; GORGES (1993), p. 8-10.

6. $A E, 1916,72=1917-1918,6$, Turgalium. D'après GOFFAUX (2006), p. 55, note 26, quoique bien moins probable, un autre développement possible serait : Anno $c$ (ivitatis) CCI/III. Toutefois, tout en adoptant le cadre temporel romain, cela serait tout aussi intéressant d'un point de vue d'affirmation identitaire face à la colonie voisine.

7. GOFFAUX (2006), p. 56-57, va même plus loin : étant donné que la juridiction d'une préfecture est exercée par les magistrats de la colonie, comme semble le suggérer le texte de Siculus Flaccus, Th., 124, 19-20, l'édile attesté à Turgalium [CIL, II, 5276 (HEp, 4, 252 ; HEp, 6, 250 ; HEp , 7, 271)] serait en réalité un magistrat de la colonie d'Augusta Emerita présent dans la praefectura.

8. Inscription malheureusement disparue aujourd'hui, ERAE, 58 (EE, VIII, 23), Augusta Emerita, indiquant le texte suivant: [[Nerone]] Claudio Caesare III co (n) s (ule),/Vitulus et Proculus Valeri fratres/Tarmest (ini ?) Lacipaea votum solver (unt) $l$ (ibentes) $m$ (erito). La mention du troisième consulat de Néron, ici victime de la damnatio memoriae, permet de la dater de 58-59.

9. $120 \mathrm{~cm}$ de large sur $21 \mathrm{~cm}$ de haut.

10. L'Itinéraire d'Antonin, 438, 4 , la situe à vingt milles d'Augusta Emerita, probablement au nordest.

11. HEp, 6, 187 (AE, 1994, 877) à Albalà del Caudillo ; HEp, 6, 249 à Torre de Santa Maria ; HEp, 6, 226 à Conquista de la Sierra, dans la praefectura de la capitale. Les variantes orthographiques sont, dans cette région, peu surprenantes.

12. ALONSO SÁNCHEZ, CERILLO M. DE CÁCERES, FERNÁNDEZ CORRALES (1994), p. 73-74 pour un point historiographique et p. 77-78 pour l'étude, qui est basée sur l'analyse d'éléments divers comme les sources littéraires, l'historiographie, la cartographie, la photographie aérienne, la télédétection, l'étude de terrain,...

13. La taille moyenne des exploitations est de 250-300 ha, avec au moins 8 sites importants retrouvés sur 2000 ha de prospection. Cf. GORGES (1990), p. 113.

14. La Sierra de S. Pedro et son prolongement à l'est, la Sierra de Montánchez. D'ailleurs, ce constat a également été conforté par l'apport des polygones de Thiessen, réajustés par les éléments de géographie physique. Cf. ALONSO SÁNCHEZ, CERILLO M. DE CÁCERES, FERNÁNDEZ CORRALES (1994), p. 73 et 80.

15. Étude contemporaine de celle de WIEGELS (1976), p. 258-284, qui prend lui aussi en considération ce paramètre.

16. Notamment deux magistrats : HAE, 761 et CIL, II, 695.

17. À ce titre, nous voudrions rappeler la prudence dont il faut faire preuve car les hypothèses sont tributaires des aléas des découvertes épigraphiques. Nonobstant, les données dont nous disposons à l'heure actuelle nous semblent néanmoins caractéristiques de certaines tendances d'un intérêt certain. 
18. L'analyse de la répartition est basée sur NAVARRo CABALLERO et RAMírEZ SÁDABA (2003), p. 248-250, travail auquel nous renvoyons pour les références exactes. Sur 83 inscriptions dénombrées, 72 proviennent de la région qui nous intéresse.

19. En ce sens, ce constat peut conforter l'hypothèse de CURCHIN (1990b), p. 270-275. : installée dans des villae, l'élite urbaine est liée et constituée de grands propriétaires terriens.

20. Afin de ne pas entrer dans les débats concernant les délimitations coloniales, seules ont été ici prises en compte les inscriptions provenant de la cité d'Augusta Emerita, mais non celles de son territoire.

21. Respectivement CIL, II, 610, 614 et CIL, II, 693, 813. Par ailleurs, aucune évergésie d'opera publica n'est pour le moment connue pour ces deux cités.

22. Plus particulièrement la carte de la répartition des villae où l'auteur essaie de délimiter des sphères d'influence urbaine, p. 98.

23. Pour un état de la question, cf. GORGES (1990), p. 93-94 et carte p. 98. On dénombre environ 340 établissements ruraux en Lusitanie à partir de diverses fouilles et études. La plupart des découvertes sont fortuites et ne sont donc pas le résultat de prospections systématiques du territoire. Par ailleurs, il est bien souvent difficile de définir leur nature exacte puisqu'on considère que $2 / 3$ seulement sont des villae. Ces données rendent délicate toute hypothèse mais cette vison partielle, voire aléatoire semble pour l'auteur d'autant plus significative : « on peut en effet penser que ce genre de découverte respecte assez bien, en proportion, la réalité des choses, dans la mesure où il $\mathrm{y}$ a effectivement plus de chances de rencontrer des sites là où ils sont nombreux que là où ils sont rares » (p. 94).

24. Metellus, proconsul de l'Ultérieure (79-71 av. J.-C.), est traditionnellement considéré comme le fondateur de la colonie.

25. La cité est généralement située à l'emplacement de l'actuelle Cáceres, mais avec pour seule et unique preuve l'inscription CIL, II, 693.

26. Attestés par Pline, HN, IV, 117 et l'Itinéraire d'Antonin, cité comme mansio de la Via de la Plata. Castra Caecilia est traditionnellement localisé à Cáceres el Viejo au nord-est de Cáceres. Toutefois, cela semble remis en cause. Cf. CADIOU (2008), p. 287-290, pour un état de la question.

27. Le terme est utilisé ici pour désigner une réalité assez floue et caractériser cette portion d'espace urbanisé, topographiquement détachée, mais non structurellement, de l'agglomération urbaine fruit de l'évolution des anciens camps.

28. CURCHIN (1985), p. 330, n5, Casar de Cáceres: L (ucius) Aemi/lius Proc/ulus vica/nis Roud (ensibus ?)/de s (uo) $f$ (aciendum) et Le Roux (1994), p. 153. Inscription difficilement datable : $\mathrm{I}^{\text {er }}-$ II $^{\mathrm{e}}$ s.

29. Pour SAquete Chamizo (2004), p. 382, cela rendrait le terrain très peu cher et expliquerait l'ampleur du territoire éméritain. À notre sens, l'éventualité de la confiscation des terres et des déplacements de population pourrait également être envisagée, sans en avoir pour le moment davantage de preuves.

30. Cf. supra : la capitale a été créée à partir d'un site inoccupé depuis le viII siècle av. J.-C.

31. Pour SAQUeTE CHAMizo (1997), p. 52-59, cette interprétation doit être nuancée et imputée à l'influence idéologique augustéenne, insistant sur la pacification et la romanisation des indigènes.

32. $A E, 1993,892=H E p, 5$, 52. L'inscription mesure $50 \times 30 \times 25 \mathrm{~cm}$ et les lettres sont de $6 \mathrm{~cm}$ de haut.

33. Tanginus est un nom local. NAVARRo CABALLERo et RAMíREZ SÁDABA (2003), p. 378-381 dénombrent uniquement 4 inscriptions hors de Lusitanie sur les 73 référencées et la plupart sont des noms uniques d'individus de condition pérégrine (p. 363-364). 


\section{RÉSUMÉS}

Symbole par excellence de l'organisation administrative romaine, la colonie est le signe tangible de l'occupation de l'espace et de son appropriation. Délimiter, partager, organiser, sont autant de jalons qui font passer des terres au statut de territoire. Or, aborder le processus de colonisation à travers le prisme géographique, c'est avant tout comprendre la manière dont les diverses sociétés qui vivent en ces lieux façonnent leur espace selon leurs propres représentations. C'est cette perception, visible dans le paysage et sensible dans le vécu, qui donne des clés de lecture et de compréhension des colonies lusitaniennes, au premier titre desquelles Augusta Emerita, et révèle l'identité même des populations qui y vivent.

The Roman colony, emblematic symbol of administrative organization, is a tangible sign of the occupation and appropriation of space. Delimitation, division, organization are very much milestones which turn the land into territory. However, approaching the colonization process through a geographical lens is, above all, understanding the way in which different societies which live in those places shape their space according to their own representations. It is this perception, visible on the landscape and perceived in the "espace vécu" which offers the keys to reading and understanding the Lusitanian colonies, at the head of which is Augusta Emerita, and is revealing of the identity of the peoples that live there.

\section{INDEX}

Mots-clés : colonie, territoire, espace, relation, frontière, société, identité, géographie, Lusitanie Keywords : colony, territory, space, frontier, society, identity, geography, Lusitania

\section{AUTEUR}

\section{SUSANA MARCOS}

Université de Bourgogne, UMR 5594 ARTeHIS ; ANHIMA - UMR 8210 